\title{
On Sequential Track Extraction within the PMHT Framework
}

\author{
Monika Wieneke and Wolfgang Koch \\ FGAN-FKIE, Neuenahrer Strasse 20, 53343 Wachtberg, Germany \\ Correspondence should be addressed to Monika Wieneke, wieneke@fgan.de
}

Received 1 April 2007; Revised 17 August 2007; Accepted 8 October 2007

Recommended by T. Luginbuhl

Tracking multiple targets in a cluttered environment is a challenging task. Probabilistic multiple hypothesis tracking (PMHT) is an efficient approach for dealing with it. Essentially PMHT is based on expectation-maximization for handling with association conflicts. Linearity in the number of targets and measurements is the main motivation for a further development and extension of this methodology. In particular, the problem of track extraction and deletion is apparently not yet satisfactorily solved within this framework. A sequential likelihood-ratio (LR) test for track extraction has been developed and integrated into the framework of traditional Bayesian multiple hypothesis tracking by Günter van Keuk in 1998. As PMHT is a multiscan approach as well, it also has the potential for track extraction. In this paper, an analogous integration of a sequential LR test into the PMHT framework is proposed. We present an LR formula for track extraction and deletion using the PMHT update formulae. The LR is thus a by-product of the PMHT iteration process, as PMHT provides all required ingredients for a sequential LR calculation. Therefore, the resulting update formula for the sequential LR test affords the development of track-before-detect algorithms for PMHT. The approach is illustrated by a simple example.

Copyright (c) 2008 M. Wieneke and W. Koch. This is an open access article distributed under the Creative Commons Attribution License, which permits unrestricted use, distribution, and reproduction in any medium, provided the original work is properly cited.

\section{INTRODUCTION}

The problem of tracking multiple targets in a realistic environment has been an object of research for a long time. The traditionalapproaches to multiple hypothesis tracking (MHT) rely on the complete enumeration of all possible associationinterpretations of a series of measurements [1]. These Bayesian MHT algorithms use a hard association model which (in the case of point targets) realistically implies that a target can produce at most one measurement at a time. A consistent realization of this model would yield an optimal tracking. Unfortunately, as the underlying problem is NP-hard, the resulting hypothesis trees grow exponentially. The so-called growing memory disaster of MHT is avoided by pruning, gating, and combining techniques which lead to an approximation of an optimal tracking. The aim is to drastically limit the number of hypotheses by retaining only the most likely ones, while the main risk is to eliminate correct measurement sequences. As a path in a hypothesis tree spans all time scans, from the past up to the present, Bayesian MHT is counted among the multiscan approaches. Another traditional approach is realized by the joint probabilistic data association filter (JPDAF) [2] that processes only the current time scan (single scan). The JPDAF is an extension of the simple PDAF for the case of multiple targets. At each scan, JPDAF combines all possible hypotheses to one synthetic hypothesis (global combining). The PDAF and JPDAF, respectively, are a second-order approximation of an optimal tracking.

A powerful, alternative approach is represented by probabilistic multiple hypothesis Tracking (PMHT) (see [3, 4]) that joins the advantages of MHT and JPDAF. PMHT works on a sliding data window (multiscan), and exploits the information of previous and following time scans in every of its kinematic state estimations. For each window position, PMHT applies the method of expectation-maximization (EM) (see $[5,6]$ ) to the underlying data. Using the language of EM the unknown associations of measurements to targets are the so called hidden variables. Then the following algorithm, known as PMHT, can be derived. For each scan of the current window, PMHT calculates one synthetic measurement from the reported measurement set (E-Step). The particular synthesis weights depend on the state estimates of the currently processed target. They represent the probability that a certain measurement belongs to this target. The synthetic measurements are then processed by a Kalman 
smoother (M-Step), which leads to improved state estimates. The new state estimates flow into the E-Step of the following iteration such that the former association weights can be corrected. For each target the E-Step and M-Step are iteratively repeated until the state estimates converge. After shifting the window the iteration process is started for the new window position. The convergence to a local maximum is guaranteed, because this property has been proven for the EM method in general. As PMHT is based on EM, its association model is soft which implies that a target can cause more than one measurement per scan. Of course a soft association model does not reflect the reality if point targets are to be tracked, but it facilitates efficient tracking algorithms. Assuming a soft association model PMHT works optimally, because the EMMethod works optimally in general.

So PMHT is a multiple target tracking algorithm of considerable theoretical elegance. Its memory wastage is linear in all parameters: window length, number of measurements, and number of targets. Working on a sliding data window, PMHT takes the information of previous and following time scans into account. Hence, as it is a multiscan approach, it has the potential for track extraction.

Unfortunately, the standard PMHT is limited to the assumption that the number of targets is constant and known in advance. Although there exist several approaches for track extraction and deletion within PMHT, this problem is apparently not yet satisfactorily solved. The most important task within a track management system is the choice of an appropriate test function for track candidates [7, 8]. Some authors [9] use statistical hypothesis testing outside PMHT to determine whether a track is true or false. Target visibility is an approach published in $[7,10,11]$. For track extraction in Bayesian MHT, a sequential likelihood-ratio (LR) test has been proposed in [12]. As this LR test has been successfully embedded into the framework of Bayesian MHT, we are motivated to try an analogous integration into the PMHT framework. In this work, we derive an LR formula for sequential track extraction by PMHT. Using this formula the LR is a by-product of the iteration process on the PMHT data window.

The remainder of this work is organized as follows. In Section 2, we provide some basics. The section begins with an introduction of our notations. Afterwards we briefly explain the method of EM and a modification of the PMHT algorithm as it is used in our work. In Section 3, we start with the principle of LR testing, as it is proposed in [12]. Then we show the derivation of an LR formula for PMHT. Section 4 presents values of the formula in an experimental example. The last section provides conclusions.

\section{PROBABILISTIC MULTIPLE HYPOTHESIS TRACKING}

To introduce our notations we start with a formal description of the considered scenario and the task of tracking multiple targets.

Our tracking scenario is defined as follows. A sensor observes $S$ point targets in its field of view (FoV). We denote the area of the FoV as $|\mathrm{FoV}|$. The sensor generates measurements $\mathbb{Z}=\mathbb{Z}_{1: T}=\left\{\mathbf{z}_{t}, N_{t}\right\}_{t=1}^{T}$ for a time interval [1:T].
The sensor output at a scan $t$ consists of not only the set of measurements $\mathbf{z}_{t}$ but also the number of measurements $N_{t}$. Thus we model measured data as a pair $\left\{\mathbf{z}_{t}, N_{t}\right\}$. Measurements $\mathbf{z}_{t}^{n} \in \mathbb{R}^{2}$ with $n \in\left[1: N_{t}\right]$ are assumed to be Cartesian position data. The spurious, noninformative measurement $n=0$ denotes a missing detection. We introduce it to avoid the hospitality problem of the standard PMHT. Its impact is explained in Section 2.3.

The task of tracking consists in estimating the kinematic states $\mathcal{X}=\mathcal{X}_{1: T}$ of the observed targets. The states $\mathbf{x}_{t}^{s} \in \mathbb{R}^{4}$ with $s \in[1: S]$ comprise position and velocity. Difficulties arise from unkown associations $\mathcal{A}=\mathcal{A}_{1: T}=\left\{\mathbf{a}_{t}\right\}_{t=1}^{T}$ of measurements to targets. We model the associations as random variables $\mathbf{a}_{t}=\left\{a_{t}^{n}\right\}_{n=0}^{N_{t}}$ that map each measurement $n \in\left[0: N_{t}\right]$ to one of the targets $s \in[0: S]$ by assigning $a_{t}^{n}=s$. The target $s=0$ is a spurious planar target that represents clutter. It corresponds to $|\mathrm{FoV}|$ and has been integrated into PMHT by [13]. So mathematically expressed, the optimization problem

$$
\arg \max _{x} p(x \mid \mathcal{Z})
$$

is to be solved. Expectation-maximization (EM) is an efficient method for this task.

\subsection{Expectation-maximization}

Expectation-maximization (EM) is an iterative method for localizing posterior modes. It has been derived and explained in many different ways. We decided to follow the work by Dellaert [5], which is one of the more descriptive derivations.

At each iteration, EM first calculates posterior weight $p\left(\mathcal{A} \mid \mathcal{Z}, \mathcal{X}^{l}\right)$. The posterior weights define an optimal lower bound

$$
\mathcal{Q}\left(\mathcal{X} ; \mathcal{X}^{l}\right)=\log p(\mathcal{X})+\sum_{\mathcal{A}} \log (p(\mathcal{A}, \mathcal{Z} \mid \mathcal{X})) p\left(\mathcal{A} \mid \mathcal{Z}, \mathcal{X}^{l}\right)
$$

of $p(\mathcal{X} \mid \mathcal{Z})$ at the current guess $\mathcal{X}^{l} . l$ is the iteration index. As $\mathcal{Q}\left(\mathcal{X} ; \mathcal{X}^{l}\right)$ is expressed as an expectation, this first step is called E-Step. In the following M-Step, EM maximizes the bound with respect to the free variable $\mathcal{X}$, which leads to improved estimates $\mathcal{X}^{(l+1)}$. They control the lower bound of the following E-Step. E-Step and M-Step are repeated until the estimates converge. How the M-Step is done depends on the application. PMHT is the application of EM to the tracking problem. It results in estimates $\mathbf{x}_{t}^{s}$ for each target $s \in[1: S]$ at each time $t \in[1: T]$. Covariance matrices $\mathbf{P}_{t}^{s}$ occur as a by-product. They cannot be proven to be the error covariance matrices of the point estimates $\mathbf{x}_{t}^{s}$, but nevertheless have a useful role.

\subsection{Calculating the posterior weights (E-Step)}

The $\mathcal{Q}$-Function contains all available information: the statistical models of the detection process, measurement process, and target dynamics. A series of calculations is required to make the information visible. We pass on deriving dynamics and sensor model and proceed directly with the formulation of the posterior weights. Because PMHT allows multiple 
measurements per target, the random variables $a_{t}^{n}$ of the associations are stochastically independent. So applying Bayes' rule yields

$$
p\left(\mathcal{A} \mid \mathcal{Z}, \mathcal{X}^{l}\right)=\prod_{t=0}^{T} \frac{\prod_{n=0}^{N_{t}} p\left(\mathbf{z}_{t}^{n} \mid \mathbf{x}_{t}^{l a_{t}^{n}}\right) p\left(a_{t}^{n} \mid N_{t}\right)}{\sum_{\mathbf{a}_{t}} \prod_{n=0}^{N_{t}} p\left(\mathbf{z}_{t}^{n} \mid \mathbf{x}_{t}^{l a_{t}^{n}}\right) p\left(a_{t}^{n} \mid N_{t}\right)}
$$

After some technical intermediate steps, that afford an exchange of product and sum in the denominator of (3), we finally obtain posterior weights

$$
\begin{aligned}
p\left(\mathcal{A} \mid \mathcal{Z}, \mathcal{X}^{l}\right) & =\prod_{t=1}^{T} \frac{\prod_{n=0}^{N_{t}} \mathcal{N}\left(\mathbf{z}_{t}^{n} ; \mathbf{H x}_{t}^{l a_{t}^{n}}, \mathbf{R}_{t}^{n}\right) \pi_{t}^{n a_{t}^{n}}}{\prod_{n=0}^{N_{t}} \sum_{s=0}^{S} \mathcal{N}\left(\mathbf{z}_{t}^{n} ; \mathbf{H} \mathbf{x}_{t}^{l s}, \mathbf{R}_{t}^{n}\right) \pi_{t}^{n s}} \\
& =: \prod_{t=1}^{T} \prod_{n=0}^{N_{t}} w_{t}^{\ln a_{t}^{n}},
\end{aligned}
$$

with $\pi_{t}^{n s}=p\left(a_{t}^{n}=s \mid N_{t}\right)$. Note that the notation (4) is simplified. With respect to the special cases $n=0$ and $s=0$, we point out that the Gaussians are to be understood in an improper sense: as clutter measurements can be assumed to be equally distributed over the FoV, the posterior weight of the clutter target $s=0$ becomes

$$
w_{t}^{\ln 0}=\sigma \cdot \frac{\pi_{t}^{n 0}}{|\mathrm{FoV}|} \quad \text { for } n>0
$$

with normalization constant $\sigma$.

And the intermediate result (3) allows us to assume

$$
w_{t}^{l 0 a_{t}^{0}}=\frac{\pi_{t}^{0 a_{t}^{0}}}{\sum_{s=0}^{S} \pi_{t}^{0 s}}=\pi_{t}^{0 a_{t}^{0}} \quad \text { for } a_{t}^{0} \in[0: S], l \in \mathbb{N}_{0} .
$$

As the posterior weights in (4) are governed by the measurement covariances $\mathbf{R}_{t}^{n}$, which is an essential characteristic trait of standard PMHT, they do not take the quality of the current track estimation into account. This problem of standard PMHT is called nonadaptivity and has already been pointed out by Willett et al. [14]. According to [15] we exchange the measurement covariances by covariances $\mathbf{S}^{\ln s}:=\mathbf{H} \mathbf{P}_{t}^{l s} \mathbf{H}^{T}+\mathbf{R}_{t}^{n}$ to make PMHT work adaptively [16]. Here $\mathbf{H}$ is the measurement matrix and $\mathbf{P}_{t}^{l s}$ is the covariance-type matrix being an output of PMHT (see Section 2.1), which is here interpreted as estimation error covariance of $\mathbf{x}_{t}^{l s}$ in the sense of a heuristic. This leads to posterior weights

$$
\begin{aligned}
p\left(\mathcal{A} \mid \mathcal{Z}, \mathcal{X}^{l}\right) & =\prod_{t=1}^{T} \frac{\prod_{n=0}^{N_{t}} \mathcal{N}\left(\mathbf{z}_{t}^{n} ; \mathbf{H} \mathbf{x}_{t}^{l a_{t}^{n}}, \mathbf{S}_{t}^{\ln a_{t}^{n}}\right) \pi_{t}^{n a_{t}^{n}}}{\prod_{n=0}^{N_{t}} \sum_{s=0}^{S} \mathcal{N}\left(\mathbf{z}_{t}^{n} ; \mathbf{H} \mathbf{x}_{t}^{l s}, \mathbf{S}_{t}^{\ln s}\right) \pi_{t}^{n s}} \\
& =: \prod_{t=1}^{T} \prod_{n=0}^{N_{t}} w_{t}^{\ln a_{t}^{n}} .
\end{aligned}
$$

The posterior weights comprise two kinds of measures that evaluate the relevance of a measurement with respect to a target estimation: a distance measure which is given by the Gaussian $\mathcal{N}\left(\mathbf{z}_{t}^{n} ; \mathbf{H x}_{t}^{l s}, \mathbf{S}^{l n s}\right)$ and a visibility measure denoted as $\pi_{t}^{n s}$. In the case of $n>0$ the latter reflects how likely it is to hit a target, not taking concrete position data into account. The weight $\pi_{t}^{0 s}$ simply is the probability of missing a target and its impact is explained in Section 2.3. In standard PMHT, $\pi_{t}^{n s}=p\left(a_{t}^{n}=s\right)$ is the association prior which is estimated iteratively by summing up the posterior weights of the current target and dividing this by the number of measurements $N_{t}[3]$. In $[7,10]$ it is proposed to estimate $\pi_{t}^{n s}$ by an HMM smoother.

We modeled the sensor output as a pair $\left\{\mathbf{z}_{t}, N_{t}\right\}$. So we can split the pair and treat $N_{t}$ separately. This leads to posteriors $\pi_{t}^{n s}:=p\left(a_{t}^{n}=s \mid N_{t}\right)$, with respect to the number of measurements $N_{t}$ in the FoV. As already proposed in [14], (Section II.C.: PMHT Implementation Issues, issue 3: Prior Probabilities) and [17], theseweights can be calculated before starting the iteration process and need not to be estimated iteratively. The calculation method is based on a valid statistical sensor model, that is the correct value is conditioned on the number of measurements $N_{t}$ received in scan $t$, and parameterized by the clutter density, by $\mid$ FoV $\mid$ and the probability of detection $P_{D}$, which is assumed to be equal for all targets. The idea behind this approach is the following: the original PMHT allows more than one measurement per target in each scan (i.e., in contrast to the physical measurement process), the calculation of $\pi_{t}^{n s}$ is an attempt to make use of the physically "correct" assignment model without destroying linearity in the number of targets. We exemplarily show the derivation via Bayes' rule for the case of $n>0$, $s>0, N_{t}>1$, and a single target $(S=1)$. For the prior we simply get $p\left(a_{t}^{n}=1\right)=P_{D} /\left(\left(1-p_{F}(0)\right)+P_{D}\right)$, whereas the denominator results from the normalization with respect to the targets. $p_{F}(0)$ denotes the probability of having no false measurements (Poisson distributed). Now we are looking for the probability of having $N_{t}$ measurements. As at most one of the measurements can be associated with the real target, the remaining measurements must be clutter. So we have $p\left(N_{t} \mid a_{t}^{n}=1\right)=p_{F}\left(N_{t}-1\right)$ and finally come to $p\left(a_{t}^{n}=s \mid N_{t}\right)$ via Bayes' rule. Further details about the calculation $\pi_{t}^{n s}$ can be found in [16]. We also derived formulae for the case of detecting the clutter target $\left(\pi_{t}^{n 0}, n>0\right)$ and missing the real target $\left(\pi_{t}^{01}\right)$.

In a scenario of multiple targets $(S>1)$ we use binomial coefficients to calculate $\pi_{t}^{n s}$. Again we show the case $n>0$ and $s>0$, that is we are looking for the probability $\pi_{t}^{n s}$ of detecting the real target. The calculation of the prior is completely analogous to the single target scenario $S=1$. Let us consider $p\left(N_{t} \mid a_{t}^{n}=s\right)$. It is given in advance that a measurement $n \in\left[1: N_{t}\right]$ refers to a real target $s \in[1: S]$. Hence, at least one real target is detected. So we have $p\left(N_{t}=0 \mid a_{t}^{n}=s\right)=0$ because there is at least one measurement. $N_{t} \in[1: S]$ measurements can be generated as follows: one measurement is given by the detection of the real target $a_{t}^{n}=s$. To generate the remaining measurements we can use another $s_{D} \in\left[0: N_{t}-1\right]$ detections of real targets. Additionally there are $\left[N_{t}-1: 0\right]$ false measurements to be produced. For the selection of a number of $s_{D}$ real targets there are $\left(\begin{array}{c}S-1 \\ s_{D}\end{array}\right)$ possibilities. The set of detectable real targets is to be reduced by the target $s$ which is already known as detected. $S-1-s_{D}$ real targets are not detected. Analogously $N_{t}>S$ measurements are generated as follows: 
one measurement arises from the given detection. Besides, another $s_{D} \in[0: S-1]$ detections of real targets can be included. Additionally $\left[N_{t}-1: N_{t}-S\right]$ false measurements have to be produced:

$$
p\left(N_{t} \mid a_{t}^{n}=s\right)=\left\{\begin{array}{l}
0, \quad N_{t}=0 \\
\sum_{s_{D}=0}^{N_{t}-1} p_{F}\left(N_{t}-s_{D}-1\right)\left(\begin{array}{c}
S-1 \\
s_{D}
\end{array}\right) \\
\times P_{D}^{s_{D}}\left(1-P_{D}\right)^{\left(S-1-s_{D}\right)}, \quad N_{t} \in[1: S] \\
\sum_{s_{D}=0}^{S-1} p_{F}\left(N_{t}-s_{D}-1\right)\left(\begin{array}{c}
S-1 \\
s_{D}
\end{array}\right) \\
\quad \times P_{D}^{s_{D}}\left(1-P_{D}\right)^{\left(S-1-s_{D}\right)}, \quad N_{t}>S .
\end{array}\right.
$$

Note $s_{D}$ does not contain the target that is already known as detected. In the case of $P_{D}=1$ there are at least $S$ measurements. Hence, we have $p\left(N_{t} \mid a_{t}^{n}=s\right)=0$ for $N_{t}<S$ and $p\left(N_{t} \mid a_{t}^{n}=s\right)=p_{F}\left(N_{t}-S\right)$ for $N_{t} \geq S$. The remaining formulae and an extensive discussion can be found in [16]. Note that the $\pi_{t}^{n s}$ have to be normalized with respect to the targets.

\subsection{Maximizing the Q-function (M-Step)}

Because the $\mathcal{Q}$-function can be rewritten as a sum

$$
\begin{aligned}
& \mathcal{Q}\left(\mathcal{X} ; \mathcal{X}^{l}\right) \\
& =\sum_{s=0}^{S}\left\{\log p\left(\mathbf{x}_{0}^{s}\right) \quad\right. \text { Initialization } \\
& +\sum_{t=1}^{T}\left\langle\log \mathcal{N}\left(\mathbf{x}_{t}^{s} ; \mathbf{F} \mathbf{x}_{t-1}^{s}, \mathbf{D}\right) \quad\right. \text { Dynamics model } \\
& \left.\left.\quad+\sum_{n=0}^{N_{t}} \log \left[\mathcal{N}\left(\mathbf{z}_{t}^{n} ; \mathbf{H x}_{t}^{s}, \mathbf{R}_{t}^{n}\right) \pi_{t}^{n s}\right] w_{t}^{l n s}\right\rangle\right\}
\end{aligned}
$$

Sensor model

over the targets, the maximization problem decomposes into $S$ independent problems: one summand per target. Let us denote one of the summands by $\mathcal{Q}^{s}\left(\mathcal{X} ; \mathcal{X}^{l}\right)$. Obviously the result of the maximization is not affected by multiplying the summand by an arbitrary constant $\alpha_{s}^{l}>0$ leading to

$$
\begin{aligned}
& \mathcal{Q}^{s}\left(\mathcal{X} ; \mathcal{X}^{l}\right) \\
& =\log p\left(\mathbf{x}_{0}^{s}\right) \alpha_{s}^{l} \quad \text { Initialization } \\
& +\sum_{t=1}^{T}\left\langle\log \mathcal{N}\left(\mathbf{x}_{t}^{s} ; \mathbf{F} \mathbf{x}_{t-1}^{s}, \mathbf{D}\right) \alpha_{s}^{l} \quad\right. \text { Dynamics model } \\
& \\
& \left.+\sum_{n=0}^{N_{t}} \log \left[\mathcal{N}\left(\mathbf{z}_{t}^{n} ; \mathbf{H} \mathbf{x}_{t}^{s}, \mathbf{R}_{t}^{n}\right) \pi_{t}^{n s}\right] w_{t}^{l n s} \alpha_{s}^{l}\right\rangle \\
& \text { Sensor model }
\end{aligned}
$$

$\alpha_{s}^{l}>0$ is constant over all scans $t$ of the current data window and all measurements $n$. It can be varied with respect to the targets $s$ and the iteration index $l$. After shifting the data window new constants $\alpha_{s}^{l}$ can be chosen. The sum over the measurements

$$
\begin{aligned}
& \sum_{n=0}^{N_{t}} \log \left[\mathcal{N}\left(\mathbf{z}_{t}^{n} ; \mathbf{H} \mathbf{x}_{t}^{s}, \mathbf{R}_{t}^{n}\right) \pi_{t}^{n s}\right] w_{t}^{\operatorname{lns}} \alpha_{s}^{l} \\
& \quad=\sum_{n=0}^{N_{t}} \log \mathcal{N}\left(\mathbf{z}_{t}^{n} ; \mathbf{H} \mathbf{x}_{t}^{s}, \mathbf{R}_{t}^{n}\right) w_{t}^{\ln s} \alpha_{s}^{l}+\text { const. }^{n}
\end{aligned}
$$

contains expressions const. ${ }^{n}:=\log \pi_{t}^{n s} w_{t}^{n s} \alpha_{s}^{l}$ with $n \in[0$ : $\left.N_{t}\right]$. As these expressions do not depend on $\mathcal{X}^{s}$, they are irrelevant for the maximization and can be ignored. Additionally we are allowed to apply the monotonically increasing exponential function, which also has no impact on the maximization result for $\mathcal{Q}^{s}\left(\mathcal{X} ; \mathcal{X}^{l}\right)$. Then for each $n$, the summand in the right part of (11) becomes

$$
\begin{aligned}
\exp ( & \left.\log \mathcal{N}\left(\mathbf{z}_{t}^{n} ; \mathbf{H} \mathbf{x}_{t}^{s}, \mathbf{R}_{t}^{n}\right) w_{t}^{\ln s} \alpha_{s}^{l}\right) \\
& =\mathcal{N}\left(\mathbf{z}_{t}^{n} ; \mathbf{H} \mathbf{x}_{t}^{s}, \mathbf{R}_{t}^{n}\right)^{w_{t}^{l n s} \alpha_{s}^{l}} \\
& \propto \frac{1}{\sqrt{\left|2 \pi \mathbf{R}_{t}^{n}\right|}} \exp \left(\boldsymbol{v}_{t}^{n s}\left(\mathbf{R}_{t}^{n}\right)^{-1} w_{t}^{l n s} \alpha_{s}^{l} \boldsymbol{v}_{t}^{n s \top}\right) \\
& \propto \mathcal{N}\left(\mathbf{z}_{t}^{n} ; \mathbf{H} \mathbf{x}_{t}^{s}, \frac{\mathbf{R}_{t}^{n}}{w_{t}^{l n s} \alpha_{s}^{l}}\right)
\end{aligned}
$$

with $\boldsymbol{v}_{t}^{n s}:=\mathbf{z}_{t}^{n}-\mathbf{H} \mathbf{x}_{t}^{s}$, the innovation of measurement $\mathbf{z}_{t}^{n}$. Starting with the $\mathcal{Q}$-function (10), we thus obtain

$$
\sum_{n=0}^{N_{t}} \log \left[\mathcal{N}\left(\mathbf{z}_{t}^{n} ; \mathbf{H} \mathbf{x}_{t}^{s}, \mathbf{R}_{t}^{n}\right) \pi_{t}^{n s}\right] w_{t}^{\ln s} \alpha_{s}^{l} \propto \prod_{n=0}^{N_{t}} \mathcal{N}\left(\mathbf{z}_{t}^{n} ; \mathbf{H} \mathbf{x}_{t}^{s}, \frac{\mathbf{R}_{t}^{n}}{w_{t}^{\ln s} \alpha_{s}^{l}}\right)
$$

for the measurement sums (over $n$ ). Analogously, with respect to the time sum (over $t$ ), we have

$$
\sum_{t=1}^{T} \log \mathcal{N}\left(\mathbf{x}_{t}^{s} ; \mathbf{F} \mathbf{x}_{t-1}^{s}, \mathbf{D}\right) \alpha_{s}^{l} \propto \prod_{t=1}^{T} \mathcal{N}\left(\mathbf{x}_{t}^{s} ; \mathbf{F} \mathbf{x}_{t-1}^{s}, \frac{\mathbf{D}}{\alpha_{s}^{l}}\right) .
$$

Successively applying the product formula (A.3) to expression (13), finally yields relation (15) with evolution matrix $\mathbf{F}$ and process noise covariance $\mathbf{D} . \overline{\mathbf{z}}_{t}^{l s}$ and $\overline{\mathbf{R}}_{t}^{l s}$ denote synthetic measurements and corresponding error covariances, respectively:

$$
\begin{aligned}
& \exp \mathcal{Q}^{s}\left(\mathcal{X}_{0: T}^{s} ; \mathcal{X}_{0: T}^{l s}\right) \\
& \propto p\left(\mathbf{x}_{0}^{s}\right)^{\alpha_{s}^{l}} \prod_{t=1}^{T} \mathcal{N}\left(\mathbf{x}_{t}^{s} ; \mathbf{F} \mathbf{x}_{t-1}^{s}, \frac{\mathbf{D}}{\alpha_{s}^{l}}\right) \mathcal{N}\left(\overline{\mathbf{z}}_{t}^{l s} ; \mathbf{H} \mathbf{x}_{t}^{s}, \overline{\mathbf{R}}_{t}^{l s}\right)
\end{aligned}
$$

with

$$
\overline{\mathbf{z}}_{t}^{l s}=\overline{\mathbf{R}}_{t}^{l s} \sum_{n=0}^{N_{t}} w_{t}^{l n s} \alpha_{s}^{l}\left(\mathbf{R}_{t}^{n}\right)^{-1} \mathbf{z}_{t}^{n}, \quad \overline{\mathbf{R}}_{t}^{l s}=\left(\sum_{n=0}^{N_{t}} w_{t}^{l n s} \alpha_{s}^{l}\left(\mathbf{R}_{t}^{n}\right)^{-1}\right)^{-1} .
$$

$\alpha_{s}^{l}$ has no influence on a synthetic measurement. Because it is constant over all measurements, it can be factored out of the 
weighted sum of measurements. Hence, as it is also contained in $\overline{\mathbf{R}}_{t}^{l s}$, it can be canceled down.

Considering the standard PMHT in a Cartesian system, that is, the case $\alpha_{s}^{l}=1$ without taking the measurement of the type $n=0$ into account and with $\mathbf{R}$ constant for all measurements, one obtains centroid measurements

$$
\overline{\mathbf{z}}_{t}^{l s}=\frac{\sum_{n=1}^{N_{t}} w_{t}^{l n s} \mathbf{z}_{t}^{n}}{\sum_{n=1}^{N_{t}} w_{t}^{\ln s}} \text { with covariances } \overline{\mathbf{R}}_{t}^{l s}=\frac{\mathbf{R}}{\sum_{n=1}^{N_{t}} w_{t}^{\ln s}} .
$$

As already pointed out in [14], the standard PMHT suffers from the so-called hospitality problem: the association weights $w_{t}^{\ln s}$ are normalized with respect to the targets. Hence, summing them up over the measurements could result in a value greater than unity, which makes the synthetic measurement covariance smaller than R. As a consequence, the standard PMHT welcomes multiple measurements as only one measurement of high accuracy.

To avoid the hospitality effect, we choose $\alpha_{s}^{l}:=1 /$ $\left(\sum_{n=0}^{N_{T}} w_{T}^{l n s}\right)$ and make use of the measurement $n=0$ representing a missing detection as follows: Because the "measurement" covariance for $n=0$ is infinitively great, it is $\left(\mathbf{R}_{t}^{0}\right)^{-1} \approx 0$, and the corresponding summands in (16) vanish. So in a Cartesian system, that is, with $\mathbf{R}$ constant for all measurements, we finally obtain centroid measurements with covariances

$$
\overline{\mathbf{R}}_{t}^{l s}=\frac{\mathbf{R}}{\alpha_{s}^{l} \sum_{n=1}^{N_{t}} w_{t}^{l n s}}, \quad \alpha_{s}^{l} \sum_{n=1}^{N_{T}} w_{T}^{l n s}=\sum_{n=1}^{N_{T}} \frac{w_{T}^{\ln s}}{\sum_{n=0}^{N_{T}} w_{T}^{\ln s}}<1 .
$$

This has an intuitive interpretation: at the latest scan $T$ of the data window, the choice of $\alpha_{s}^{l}$ leads to a renormalization of the assignment weights $w_{T}^{\text {lns }}$. It enforces the sum in the denominator of (18) to be less than unity and hence mitigates the hospitality problem at the head of the data window. The posterior weight $w_{T}^{l 0 s}$ is given by $\pi_{T}^{0 s}$ (see Section 2.2), which is the probability of missing the target. Note that the integration of $\alpha_{s}^{l}$ only has an impact on the synthetic measurement covariances $\overline{\mathbf{R}}_{t}^{l_{s}}$ and not on the synthetic measurements $\overline{\mathbf{z}}_{t}^{l s}$. It must be pointed out that for elapsed scans $t=1, \ldots, T-1$, this choice of $\alpha_{s}^{l}$ does not lead to a renormalization with respect to the measurements and that at these scans the hospitality problem is possible and can even be increased. But in the past hospitality effects have a good chance to be corrected by the Kalman retrodiction (Rauch-Tung-Striebel recursion). The most sensitive PMHT estimation is at the head of the data window, where our approach avoids hospitality.

The above considerations make clear that the PMHT method of estimating $\mathcal{X}^{s}$ for each target is invariant under the replacement $\mathbf{R}_{t}^{n} \rightarrow \mathbf{R}_{t}^{n} / \alpha_{s}^{l}$ and $\mathbf{D} \rightarrow \mathbf{D} / \alpha_{s}^{l}$. The arbitrary constant $\alpha_{s}^{l}$ is therefore an internal degree of freedom inherent to PMHT. The standard formulation assumes $\alpha_{s}^{l}=1$, for all $s, l$. However, any other choice is legitimate, which affords a multitude of PMHT variants.

Now let us return to the formulation of the PMHT algorithm. The expression (15) is maximized by an ordinary
Kalman smoother that processes the synthetic values. As a result we get improved state estimates that flow into the following E-Step. So for each target, the data of the current PMHT window is processed as follows.

\section{(1) Expectation-step: calculation of posterior weight $w_{t}^{\text {lns }}$}

The weights are calculated for all scans of the current window position. They are based on the measurements $\mathbf{z}_{t}^{n}$ and the state estimations $\mathbf{x}_{t}^{l s}$. Afterwards these weights are used to calculate the synthetic measurement $\overline{\mathbf{z}}_{t}^{l_{s}}$ and corresponding error covariances $\overline{\mathbf{R}}_{t}^{l s}$.

\section{(2) Maximization-step: application of a Kalman smoother}

Using the synthetic values of the E-Step, a Kalman filter is applied to the data window. The following retrodiction yields new, improved estimation $\mathbf{x}_{0: T}^{(l+1) s}$.

After convergence, the prediction $\mathbf{x}_{T+1 \mid T}^{s}$ is to be calculated for the following window position. When all targets have been processed, the window is shifted by one scan.

\section{SEQUENTIAL TRACK EXTRACTION BY PMHT}

We need a technique that extracts the tracks of an unknown number of targets in the FoV. This should happen as fast as possible and as reliably as requested. Compared with the state estimation in track maintenance, the required algorithm works on a higher level of abstraction, that is, we are not looking for single target states but for whole tracks. A sequential likelihood-ratio (LR) test is a technique that analyzes the inflowing measurements with this objective.

\subsection{Likelihood ratio testing}

In [12] a sequential LR test has been integrated into the Bayesian MHT of well separated targets. Thereby the extraction of a track is modeled as a decision between two competing hypotheses $H_{0}$ and $H_{1}$. Referring to the given series of measurements $\mathcal{Z}_{1: t}$, they have the following meanings:

$H_{1}$ : the series $\mathbb{Z}_{1: t}$ contains data from the target and possibly clutter;

$H_{0}$ : no target exists, hence all data in $\mathbb{Z}_{1: t}$ are false.

The aim is to decide as fast as possible and as reliably as requested between $H_{1}$ and $H_{0}$. A sequential LR test consists in successively updating the ratio $\operatorname{LR}_{1}(t)(19)$ between the two likelihood functions $p\left(H_{1} \mid \mathcal{Z}_{1: t}\right)$ and $p\left(H_{0} \mid \mathcal{Z}_{1: t}\right)$ :

$$
\operatorname{LR}_{1}(t)=\frac{p\left(\mathcal{Z}_{1: t} \mid H_{1}\right)}{p\left(\mathcal{Z}_{1: t} \mid H_{0}\right)}=\frac{p\left(\mathbf{z}_{t} \mid \mathbb{Z}_{1: t-1}, H_{1}\right)}{p\left(\mathbf{z}_{t} \mid \mathcal{Z}_{1: t-1}, H_{0}\right)} \cdot \operatorname{LR}(t-1) .
$$

At each scan $t$ the value $\operatorname{LR}_{1}(t)$ is compared with two thresholds $A$ and $B$.

(i) If $\operatorname{LR}_{1}(t) \leq A$, hypothesis $H_{0}$ is accepted to be true.

(ii) If $\operatorname{LR}_{1}(t) \geq B$, hypothesis $H_{1}$ is accepted to be true.

(iii) Otherwise the algorithm cannot come to a decision yet and has to wait for the measurements $\mathbf{z}_{t+1}$ of the next scan to test $\mathrm{LR}_{1}(t+1)$. 
This general scheme was first proposed by Wald [18]. The user has to preset the reliability of the algorithm by determining the thresholds $A$ and $B$. Thereto they have to set the related statistical decision errors $P_{1}:=\operatorname{Prob}\left(\right.$ accept $\left.H_{1} \mid H_{1}\right)$ and $P_{0}:=\operatorname{Prob}\left(\right.$ accept $\left.H_{1} \mid H_{0}\right) . P_{1}$ is the probability to rightly identify a really existing target as a target, whereas $P_{0}$ is the probability to wrongly assume the existence of a target that does not exist. The thresholds $A$ and $B$ depend on the errors $P_{1}$ and $P_{0}$ as follows:

$$
A \approx \frac{1-P_{1}}{1-P_{0}}, \quad B \approx \frac{P_{1}}{P_{0}} .
$$

The smaller the permitted error, the longer the user has to wait for the decision. For example, if $P_{1}$ is chosen near unity and $P_{0}$ is chosen near zero (corresponding to a certainty near $100 \%$ ), the runtime would by infinitively long. If the decision is requested immediately, all possible combinations of measurements will be identified as targets.

The main result of [12] is the derivation of $\operatorname{LR}_{1}(t)$ as a sum over the (not normalized) weights of all possible interpretations of $\mathbb{Z}_{1: t}$. An interpretation corresponds to a path from the root to a leaf of the hypothesis tree. This allows a seamless transition into the phase of track maintenance.

\subsection{Likelihood-ratio calculation by PMHT}

As the LR test has been successfully embedded into the framework of Bayesian MHT, we are motivated to integrate it into PMHT in an analogous manner. Like Bayesian MHT, the PMHT counts among the multiscan approaches and hence complies with the requirements of such an integration. This section shows how the LR is calculated by PMHT as a byproduct.

The following derivation relies on the assumption, that either $S$ targets reside in the FoV or none. Accordingly we define hypotheses $H_{S}$ and $H_{0}$ as follows:

$H_{S}$ : the series $\mathfrak{Z}_{1: t}$ contains data from $S$ targets and possibly clutter;

$H_{0}$ : no targets exist, hence all data in $\mathbb{Z}_{1: t}$ are false.

Assumption: $H_{S}$ and $H_{0}$ exclude each other.

As the sensor output is modeled as a pair $\left\{\mathbf{z}_{t}, N_{t}\right\}$, we can split it and treat $N_{t}$ separately. So (19) leads to the following equation:

$$
\begin{aligned}
\operatorname{LR}_{S}(t)= & \frac{p\left(\mathbb{Z}_{1: t} \mid H_{S}\right)}{p\left(\mathbb{Z}_{1: t} \mid H_{0}\right)}=\underbrace{\frac{p\left(\mathbf{z}_{t} \mid N_{t}, \mathbb{Z}_{1: t-1}, H_{S}\right)}{p\left(\mathbf{z}_{t} \mid N_{t}, \mathbb{Z}_{1: t-1}, H_{0}\right)}}_{F_{1}} \\
& \cdot \underbrace{\frac{p\left(N_{t} \mid H_{S}\right)}{p\left(N_{t} \mid H_{0}\right)}}_{F_{2}} \cdot \frac{p\left(\mathbb{Z}_{1: t-1} \mid H_{S}\right)}{p\left(\mathbb{Z}_{1: t-1} \mid H_{0}\right)} .
\end{aligned}
$$

The key idea on adopting van Keuk's sequential LR test is a new formulation of the hypotheses $H_{S}$ and $H_{0}$. That is, in factor $F_{1}$ of $(21), H_{S}$ and $H_{0}$ are defined by using the detection probability $P_{D}$ as follows:

$$
\begin{aligned}
& H_{S} \equiv H_{S} \wedge\left(P_{D} \gg 0\right), \\
& H_{0} \equiv H_{S} \wedge\left(P_{D} \approx 0\right) .
\end{aligned}
$$

The decision between $S$ and zero targets is now completely controlled by $P_{D}$ (assumed to be equal for all targets). The probabilities in factor $F_{2}$ of (21) can be easily calculated. The numerator can be written as

$$
\begin{gathered}
p\left(N_{t} \mid H_{S}\right)=\sum_{s} p\left(N_{t} \mid a_{t}^{n}=s\right) \quad \text { with } \\
n \in\left[0: N_{t}\right] \text { arbitrary, but fixed. }
\end{gathered}
$$

The summands $p\left(N_{t} \mid a_{t}^{n}=s\right)$ are the visibility weights that have been introduced and briefly explained in Section 2.2. The denominator represents the probability of having $N_{t}$ false measurements at scan $t$, which can be modeled by a Poisson distribution. We denote it as $p_{F}\left(N_{t}\right)$. So we finally get

$$
\begin{aligned}
\operatorname{LR}_{S}(t)= & \frac{p\left(\mathbf{z}_{t} \mid N_{t}, \mathcal{Z}_{1: t-1}, H_{S}, P_{D} \gg 0\right)}{p\left(\mathbf{z}_{t} \mid N_{t}, \mathcal{Z}_{1: t-1}, H_{S}, P_{D} \approx 0\right)} \\
& \cdot \frac{p\left(N_{t} \mid H_{S}\right)}{p_{F}\left(N_{t}\right)} \cdot \operatorname{LR}_{S}(t-1) .
\end{aligned}
$$

The PMHT algorithm works on the basis of synthetic measurements. Let $l$ be the number of the current PMHT iteration and $s \in[1: S]$ one of the targets. At each time step $t$, the processing of multiple measurements $\mathbf{z}_{t}^{0}, \ldots, \mathbf{z}_{t}^{N_{t}}$ is put down to the processing of a single measurement $\overline{\mathbf{z}}_{t}^{l_{s}}$. Thus in the sequential LR calculation by PMHT, we follow that principle and consider the ratio between the likelihood functions with synthetic measurements

$$
\begin{aligned}
\operatorname{LR}_{S}(t) “= & \underbrace{\frac{p\left(\overline{\mathbf{z}}_{t} \mid N_{t}, \mathcal{Z}_{1: t-1}, H_{S}, P_{D} \gg 0\right)}{p\left(\overline{\mathbf{z}}_{t} \mid N_{t}, \mathfrak{Z}_{1: t-1}, H_{S}, P_{D} \approx 0\right)}}_{F_{1}} \\
& \cdot \frac{p\left(N_{t} \mid H_{S}\right)}{p_{F}\left(N_{t}\right)} \cdot \operatorname{LR}_{S}(t-1),
\end{aligned}
$$

which is a plausible heuristic approximation of (24). Thereby the vector $\overline{\mathbf{z}}_{t}:=\left(\overline{\mathbf{z}}_{t}^{1}, \ldots, \overline{\mathbf{z}}_{t}^{S}\right)$ denotes the synthetic measurements of all targets at scan $t$ after the last iteration (on the window that ends at scan $t$ ).

In the following, we consider only the numerator of $F_{1}$ in (25) and continue by including the target states $\mathbf{x}_{t}$ via marginalization (26). Then assuming that target states are stochastically independent, we come to the product:

$$
\begin{aligned}
p\left(\overline{\mathbf{z}}_{t} \mid\right. & \left.N_{t}, \mathbb{Z}_{1: t-1}, H_{S}, P_{D} \gg 0\right) \\
& =\int p\left(\overline{\mathbf{z}}_{t}, \mathbf{x}_{t} \mid N_{t}, \mathbb{Z}_{1: t-1}, H_{S}, P_{D} \gg 0\right) d \mathbf{x}_{t} \\
& =\prod_{s=1}^{S} \int p\left(\overline{\mathbf{z}}_{t}^{s}, \mathbf{x}_{t}^{s} \mid N_{t}, \mathbb{Z}_{1: t-1}, H_{S}, P_{D} \gg 0\right) d \mathbf{x}_{t}^{s} .
\end{aligned}
$$

We proceed by considering a single factor of (27). For the sake of simplicity we forego the notation of $P_{D} \gg 0$. A factor corresponds to a target $s \in[1: S]$. Let $d_{t}^{s}$ be the detection 
state of the target $d_{t}^{s} \equiv$ detected, $\neg d_{t}^{s} \equiv$ not detected). After marginalization over $d_{t}^{s}$ we get

$$
\begin{aligned}
& \int p\left(\overline{\mathbf{z}}_{t}^{s}, \mathbf{x}_{t}^{s} \mid N_{t}, \mathbb{Z}_{1: t-1}, H_{S}\right) d \mathbf{x}_{t}^{s} \\
& =\int\left\langle p\left(\overline{\mathbf{z}}_{t}^{s}, \mathbf{x}_{t}^{s}, d_{t}^{s} \mid N_{t}, \mathcal{Z}_{1: t-1}, H_{S}\right)\right. \\
& \left.+p\left(\overline{\mathbf{z}}_{t}^{s}, \mathbf{x}_{t}^{s}, \neg d_{t}^{s} \mid N_{t}, \boldsymbol{Z}_{1: t-1}, H_{S}\right)\right\rangle d \mathbf{x}_{t}^{s} \\
& =\int\left\langle p\left(\overline{\mathbf{z}}_{t}^{s}, \mathbf{x}_{t}^{s} \mid d_{t}^{s}, N_{t}, \mathcal{Z}_{1: t-1}, H_{S}\right)\right. \\
& \times \underbrace{p\left(d_{t}^{s} \mid N_{t}, \mathbb{Z}_{1: t-1}, H_{S}\right)}_{=: \pi_{t}^{d s}} \\
& +p\left(\overline{\mathbf{z}}_{t}^{s}, \mathbf{x}_{t}^{s} \mid \neg d_{t}^{s}, N_{t}, \mathbb{Z}_{1: t-1}, H_{S}\right) \\
& \times \underbrace{p\left(\neg d_{t}^{s} \mid N_{t}, \mathbb{Z}_{1: t-1}, H_{S}\right)}_{=: \pi_{t}^{\urcorner d s}}\rangle d \mathbf{x}_{t}^{s} .
\end{aligned}
$$

The terms $\pi_{t}^{d s}$ and $\pi_{t}^{\neg d s}$ represent the detection probability of a target, given the number of measurements $N_{t}$. And they are somewhat similar to the visibility weights $\pi_{t}^{n s}=p\left(a_{t}^{n}=\right.$ $\left.s \mid N_{t}\right)$ in Section 2.2. But $\pi_{t}^{n s}$ is normalized with respect to the targets $s \in[0: S]$. In (28) we consider a fixed target $s$, that is, one of the factors in (27) and marginalize over the targets detection state $d_{t}^{s}$. Such a marginalization requires a normalization with respect to the measurements, that is, for $n>0$ and $s>0$ we have $\pi_{t, \text { renorm }}^{n s}=\pi_{t}^{n s} /\left(\pi_{t}^{0 s}+N_{t} \cdot \pi_{t}^{n s}\right)$ because $\pi_{t}^{n s}=p\left(a_{t}^{n}=s \mid N_{t}\right)$ are the same for all real measurements $n \in\left[1: N_{t}\right]$ in scan $t$ :

$$
\begin{aligned}
\pi_{t}^{\neg d s} & =p\left(\neg d_{t}^{s} \mid N_{t}, \mathbb{Z}_{1: t-1}, H_{S}\right)=\pi_{t, \text { renorm }}^{0 s} \\
\pi_{t}^{d s} & =p\left(d_{t}^{s} \mid N_{t}, \mathbb{Z}_{1: t-1}, H_{S}\right)=N_{t} \cdot \pi_{t, \text { renorm }}^{n s}
\end{aligned}
$$

Furthermore, $\pi_{t}^{d s}$ and $\pi_{t}^{\neg d s}$ are independent of the integration variable $\mathbf{x}_{t}^{s}$. Thus

$$
\begin{aligned}
=\pi_{t}^{d s} \int & \langle\underbrace{p\left(\overline{\mathbf{z}}_{t}^{s} \mid \mathbf{x}_{t}^{s}, d_{t}^{s}, N_{t}, \mathfrak{Z}_{1: t-1}, H_{S}\right)}_{D_{1}} \\
& \times \underbrace{p\left(\mathbf{x}_{t}^{s} \mid d_{t}^{s}, N_{t}, \mathfrak{Z}_{1: t-1}, H_{S}\right)}_{D_{2}}\rangle d \mathbf{x}_{t}^{s} \\
+\pi_{t}^{\neg d s} \int & \underbrace{\left.\left\langle\overline{\mathbf{z}}_{t}^{s}\right| \mathbf{x}_{t}^{s}, \neg d_{t}^{s}, N_{t}, \mathbb{Z}_{1: t-1}, H_{S}\right)}_{D_{3}} \\
& \times \underbrace{p\left(\mathbf{x}_{t}^{s} \mid \neg d_{t}^{s}, N_{t}, \mathbb{Z}_{1: t-1}, H_{S}\right)}_{D_{4}}\rangle d \mathbf{x}_{t}^{s} .
\end{aligned}
$$

The probabilities $D_{1}$ and $D_{2}$ refer to the case of detecting the target $s . D_{1}$ is the likelihood function $p\left(\overline{\mathbf{z}}_{t}^{s} \mid \mathbf{x}_{t}^{s}, d_{t}^{s}, H_{S}\right)$ of $\mathbf{x}_{t}^{s}$. It is assumed to be Gaussian: $\mathcal{N}\left(\overline{\mathbf{z}}_{t}^{s} ; \mathbf{H x}_{t}^{s}, \overline{\mathbf{R}}_{t}^{s}\right)$. In $D_{2}$ the state $\mathbf{x}_{t}^{s}$ is dependent of the measurements $\mathcal{Z}_{1: t-1}$ of elapsed scans. So for the current scan $t$, the whole information of measurements is contained in the prediction $\mathbf{x}_{t \mid t-1}^{s}$. As for the current scan the measuring information is not given, the variables $d_{t}^{s}$ and $N_{t}$ have no impact. So it makes sense to model $p\left(\mathbf{x}_{t}^{s} \mid \mathcal{Z}_{1: t-1}, H_{S}\right)$ as a Gaussian $\mathcal{N}\left(\mathbf{x}_{t}^{s} ; \mathbf{x}_{t \mid t-1}^{s}, \mathbf{P}_{t \mid t-1}^{s}\right)($ see $(31)$

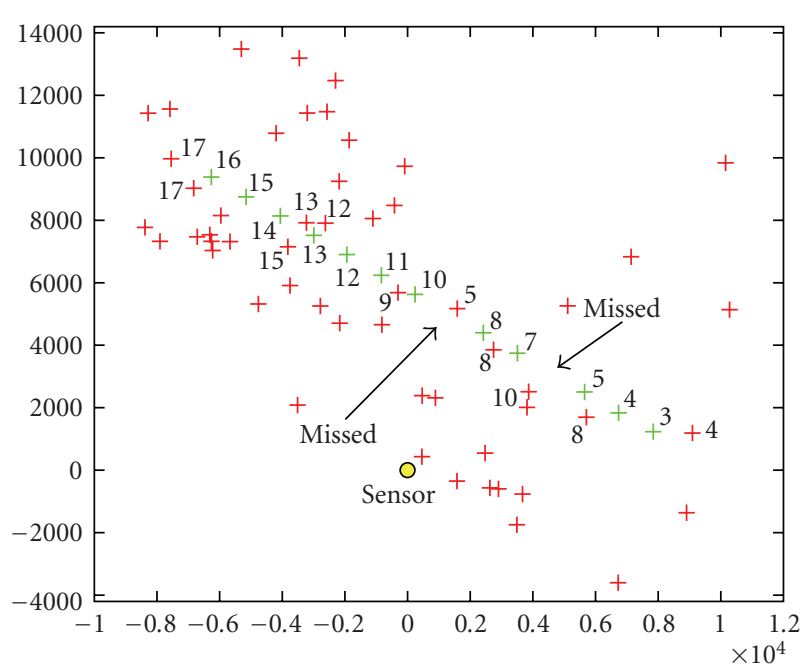

Figure 1: Movement of an aircraft along a straight line.

1st summand). The probabilities $D_{3}$ and $D_{4}$ refer to the case of missing the target. If the target has not been detected, $D_{3}$ is not constant. On every unit of the area $|\mathrm{FoV}|, \overline{\mathbf{z}}_{t}^{s}$ can be found with equal probability $p\left(\overline{\mathbf{z}}_{t}^{s} \mid \mathbf{x}_{t}^{s}, \neg d_{t}^{s}, H_{S}\right)=1 / \mid$ FoV $\mid . D_{4}$ stays below the integral and vanishes because of the normalization property (31), 2nd summand).

Using the product formula (A.1), (31) can be transformed into (32):

$$
\begin{gathered}
\int p\left(\overline{\mathbf{z}}_{t}^{s}, \mathbf{x}_{t}^{s} \mid N_{t}, \mathbf{Z}_{1: t-1}, H_{S}\right) d \mathbf{x}_{t}^{s} \\
=\ldots \\
=\pi_{t}^{d s} \int\left\langle\mathcal{N}\left(\overline{\mathbf{z}}_{t}^{s} ; \mathbf{H} \mathbf{x}_{t}^{s}, \overline{\mathbf{R}}_{t}^{s}\right) \mathcal{N}\left(\mathbf{x}_{t}^{s} ; \mathbf{x}_{t \mid t-1}^{s}, \mathbf{P}_{t \mid t-1}^{s}\right)\right\rangle d \mathbf{x}_{t}^{s}(31) \\
\quad+\pi_{t}^{\neg d s} \frac{1}{|\mathrm{FoV}|} \int p\left(\mathbf{x}_{t}^{s} \mid \ldots\right) d \mathbf{x}_{t}^{s} \\
=\pi_{t}^{d s} \mathcal{N}(\overline{\mathbf{z}}_{t}^{s} ; \mathbf{H} \mathbf{x}_{t \mid t-1}^{s}, \underbrace{\left.\mathbf{H P}_{t \mid t-1}^{s} \mathbf{H}^{\top}+\overline{\mathbf{R}}_{t}^{s}\right)}_{=: \overline{\mathbf{S}}_{t}^{s}} \int \mathcal{N}\left(\mathbf{x}_{t}^{s} ; \ldots\right) d \mathbf{x}_{t}^{s} \\
\quad+\pi_{t}^{\neg d s} \frac{1}{|\mathrm{FoV}|} .
\end{gathered}
$$

Thereby $\overline{\mathbf{S}}_{t}^{s}$ is the synthetic innovation covariance after the last PMHT iteration. Inserting (32) into (27) yields the following expression for factor $F_{1}$ of (25):

$$
\begin{aligned}
& \frac{p\left(\overline{\mathbf{z}}_{t} \mid N_{t}, \mathcal{Z}_{1: t-1}, H_{S}, P_{D} \gg 0\right)}{p\left(\overline{\mathbf{z}}_{t} \mid N_{t}, \mathcal{Z}_{1: t-1}, H_{S}, P_{D} \approx 0\right)} \\
& =\prod_{s=1}^{S} \underbrace{\frac{\pi_{t}^{d s} \mathcal{N}\left(\overline{\mathbf{z}}_{t}^{s} ; \mathbf{H x}_{t \mid t-1}^{s}, \overline{\mathbf{S}}_{t}^{s}\right)+\pi_{t}^{\pi_{t, P_{D} \approx 0}}}{\pi_{t s}^{d s}} \mathcal{N}(1 /|\mathrm{FoV}|)}_{\approx 0} .
\end{aligned}
$$



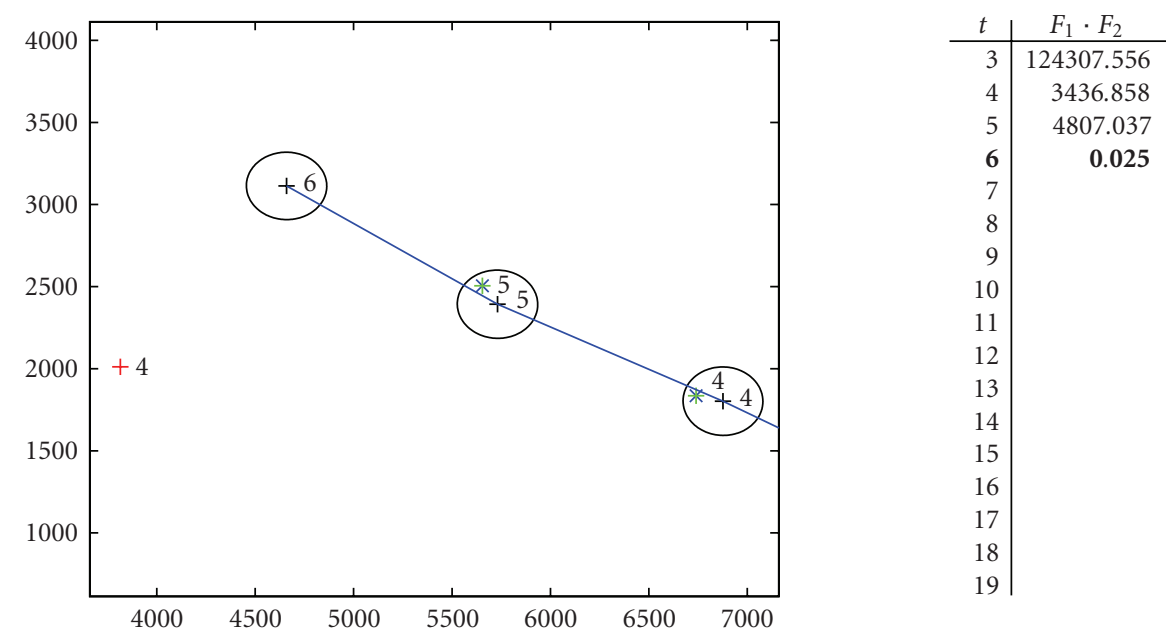

(a)
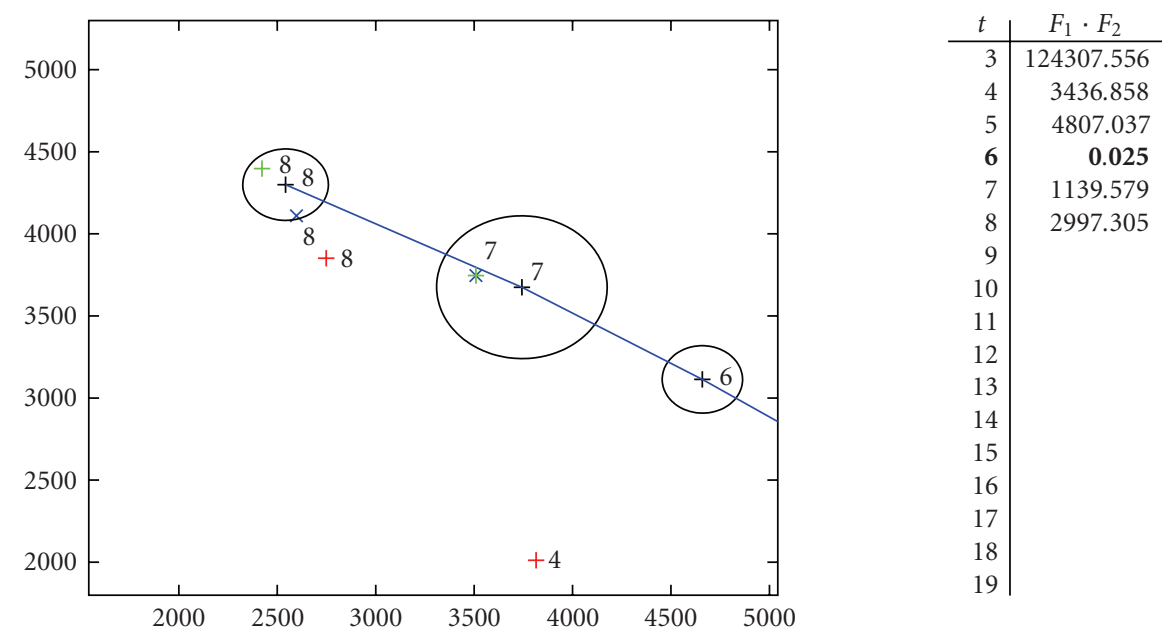

(b)

Figure 2: Missing detection $(t=6)$, aftereffect $(t=7)$, clutter $(t=8)$.

The hypothesis $H_{0}$ is expressed by $H_{S} \wedge\left(P_{D} \approx 0\right)$. In the case of no targets we have $\pi_{t}^{d s} \approx 0$ and $\pi_{t}^{\neg d s} \approx 1$. So (33) and (25) yield our final LR formua:

$$
\begin{aligned}
\operatorname{LR}_{S}(t) \propto & \underbrace{\prod_{s=1}^{S}\left(\pi_{t}^{d s} \mathcal{N}\left(\overline{\mathbf{z}}_{t}^{s} ; \mathbf{H x}_{t \mid t-1}^{s}, \overline{\mathbf{S}}_{t}^{s}\right) \cdot|\mathrm{FoV}|+\pi_{t}^{\neg d s}\right)}_{F_{1}} \\
& \underbrace{\frac{p\left(N_{t} \mid H_{S}\right)}{p_{F}\left(N_{t}\right)} \cdot \operatorname{LR}_{S}(t-1) .}_{F_{2}}
\end{aligned}
$$

Note that all ingredients of our LR formula are provided by PMHT. Thus the LR calculation (34) is a by-product of the PMHT iteration process.

\subsection{Extracting a target cluster by PMHT}

Sequential LR testing can well be extended to the problem of extracting target clusters with an unknown number of targets involved $[12,19]$. To this end assume that the number $K$ of targets involved in a cluster is limited by $K_{\max }$ (not too large). The ratio of the probability $p\left(H_{1} \vee H_{2} \cdots \vee H_{K} \mid \mathbb{Z}_{1: t}\right)$ that a cluster consisting of at least one and at most $K$ targets exists, versus the probability of having false returns only, can be written as

$$
\begin{aligned}
\frac{p\left(H_{1} \vee \cdots \vee H_{K} \mid \mathcal{Z}_{1: t}\right)}{p\left(H_{0} \mid \mathcal{Z}_{1: t}\right)} & =\frac{\sum_{n=1}^{K} p\left(H_{n} \mid \mathcal{Z}_{1: t}\right)}{p\left(H_{0} \mid \mathcal{Z}_{1: t}\right)} \\
& =\sum_{n=1}^{K} \frac{p\left(\mathcal{Z}_{1: t} \mid H_{n}\right)}{p\left(\mathcal{Z}_{1: t} \mid H_{0}\right)} \frac{p\left(H_{n}\right)}{p\left(H_{0}\right)}
\end{aligned}
$$

We thus obtain in a natural way a generalized test function $\operatorname{LR}_{K}(t)=(1 / K) \sum_{n=1}^{k} \operatorname{LR}_{n}(t)$ with $\operatorname{LR}_{n}(t)=$ $p\left(\mathbb{Z}_{1: t} \mid H_{n}\right) / p\left(\mathbb{Z}_{1: t} \mid H_{0}\right)$ to be calculated in analogy to the case $n=1$. In practical application the finite resolution capabilities of the sensors involved have to be taken into account [20]. It seems to be reasonable to interpret the normalized individual likelihood-ratios $\operatorname{LR}_{n}(t) / \sum_{n=1}^{K} \operatorname{LR}_{n}(t)=c_{t}(n)$ as a "cardinality," that is as a measure of the probability of having $n$ objects in the cluster. An estimator for the number of 

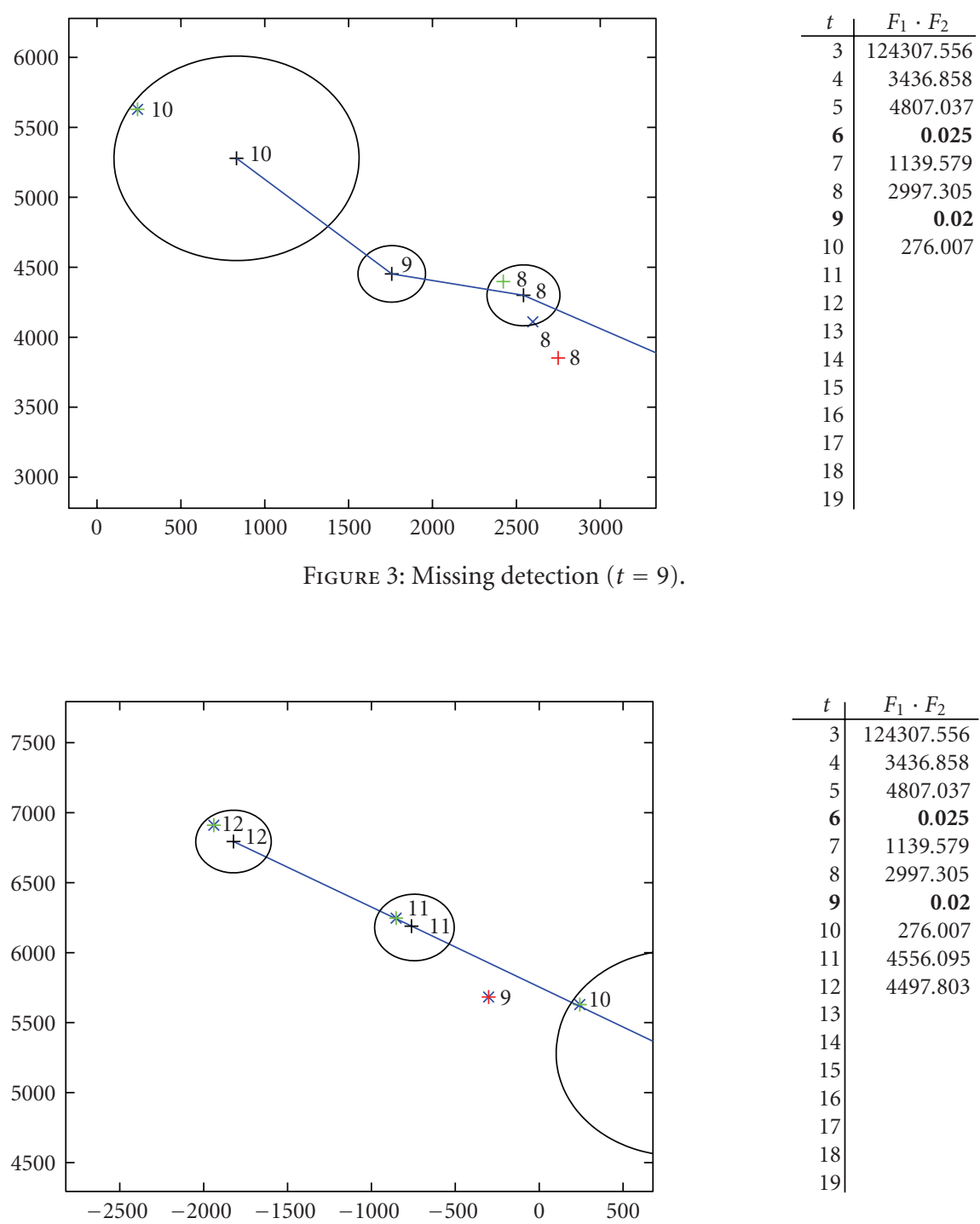

(a)
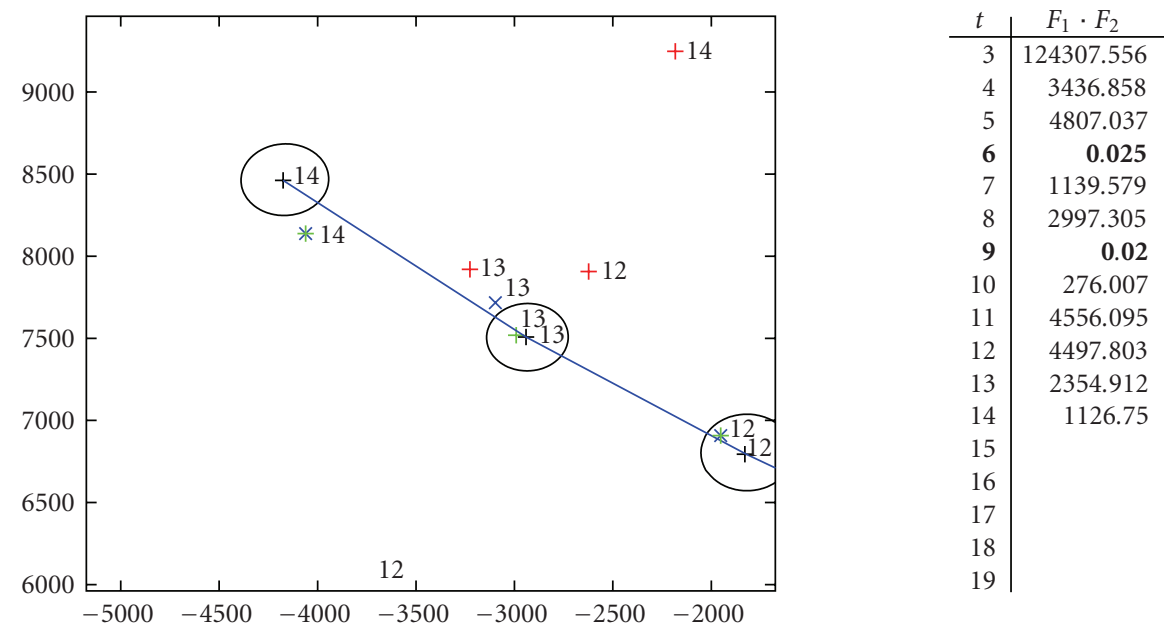

(b)

FIgURE 4: Stable tracking $(t=11,12)$ and impact of clutter $(t=13)$. 

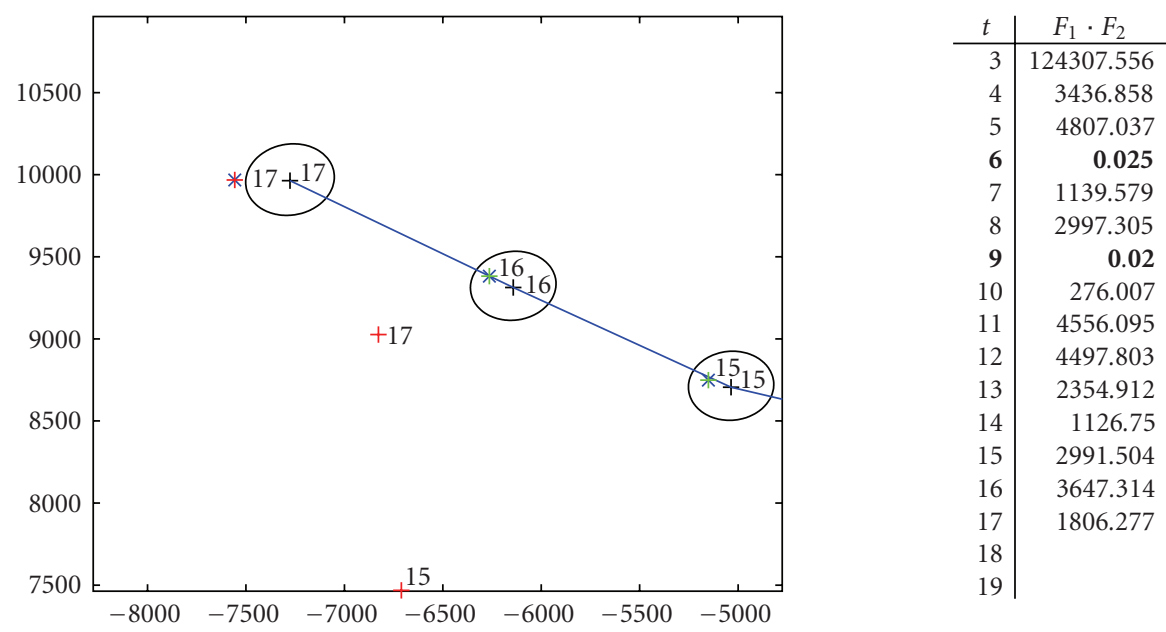

(a)

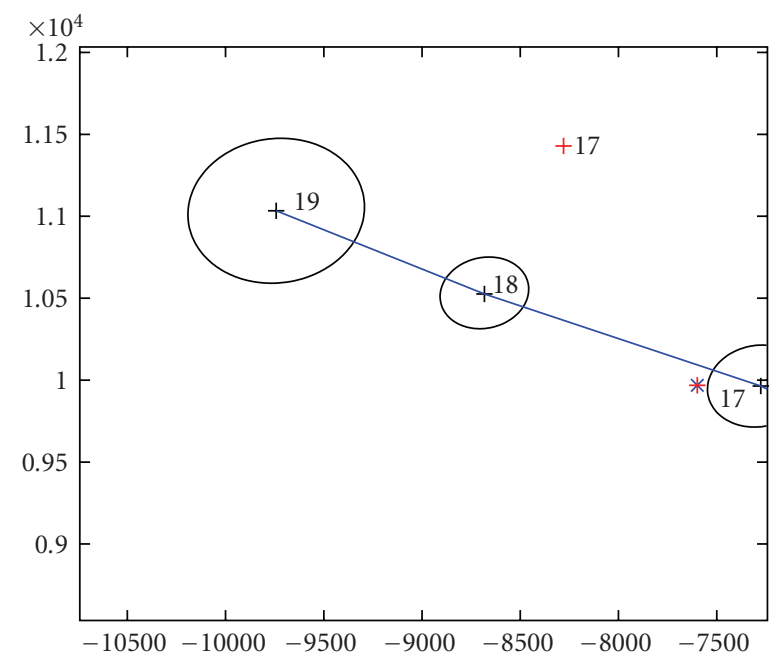

(b)

\begin{tabular}{r|r}
\multicolumn{1}{c|}{$t$} & \multicolumn{1}{|c}{$F_{1} \cdot F_{2}$} \\
\hline 3 & 124307.556 \\
4 & 3436.858 \\
5 & 4807.037 \\
$\mathbf{6}$ & $\mathbf{0 . 0 2 5}$ \\
7 & 1139.579 \\
8 & 2997.305 \\
$\mathbf{9}$ & $\mathbf{0 . 0 2}$ \\
10 & 276.007 \\
11 & 4556.095 \\
12 & 4497.803 \\
13 & 2354.912 \\
14 & 1126.75 \\
15 & 2991.504 \\
16 & 3647.314 \\
17 & 1806.277 \\
$\mathbf{1 8}$ & $\mathbf{0 . 0 1 9}$ \\
$\mathbf{1 9}$ & $\mathbf{0 . 0 8 1}$
\end{tabular}

FIGURE 5: Vanishing of the aircraft at scan $t=17$.

targets within the cluster is thus given by $\bar{n}=\sum_{n=1}^{K} n c(n)$. Using the results of Section 3.2, (35) can also be evaluated within the PMHT framework.

\section{EXPERIMENTAL EXAMPLE}

This section shows the values of the product $F_{1} \cdot F_{2}$ during the tracking. We simulated a simple scenario with one target. A rotating radar observes an aircraft in its FoV. The total length of observation is 25 scans. The aircraft moves along a straight line. The movement starts at scan 1 and ends at scan 16 . Since scan 17 we generated false measurements only. The distance $\Delta t$ between two consecutive scans is 5 seconds (time of circulation). False measurements are generated with a density $\rho_{F}=10^{-7.2}$ (in events per $\mathrm{m}^{2}$ ). For the aircraft we assumed a detection probability $P_{D}=0.8$. Figure 1 shows the measurements of scan 3 up to scan 17. The distance labels on the axes refer to meters. The plot shows real measurements as green crosses + , labeled by scan numbers. False alarms are marked as red crosses + . They are plotted only within a ra- dius of $3000 \mathrm{~m}$ around the true position. At the scans $t=6$ and $t=9$ the aircraft was not detected.

\subsection{Implementation issue}

Starting with a window length of 3 , we let the PMHT window grow up to a length of 7 scans and shifted it (by one scan) until the head reached scan 25 . At each window position 7 EM iterations were processed. In the following figures, we use black color $(+)$ for the prediction $\mathbf{x}_{t}^{s}$ and its error ellipsoid. The particular synthetic measurement $\overline{\mathbf{z}}_{t}^{s}$ is noted as a blue cross $\times$.

From a formalistic point of view, the parameter $\alpha_{s}^{l}$ has to be constant over all scans $t$ of the current data window and all measurements $n$. During our experiments we found out that the results could be improved using a time-adaptive parameter $\alpha_{s}^{l}(t)$ that varies over the scans inside the data window. Choosing $\alpha_{s}^{l}(t)=1 /\left(\sum_{n=0}^{N_{t}} w_{t}^{\text {lns }}\right)$, hospitality is avoided at all scans of the current data window. The following results have been generated with this extension. 


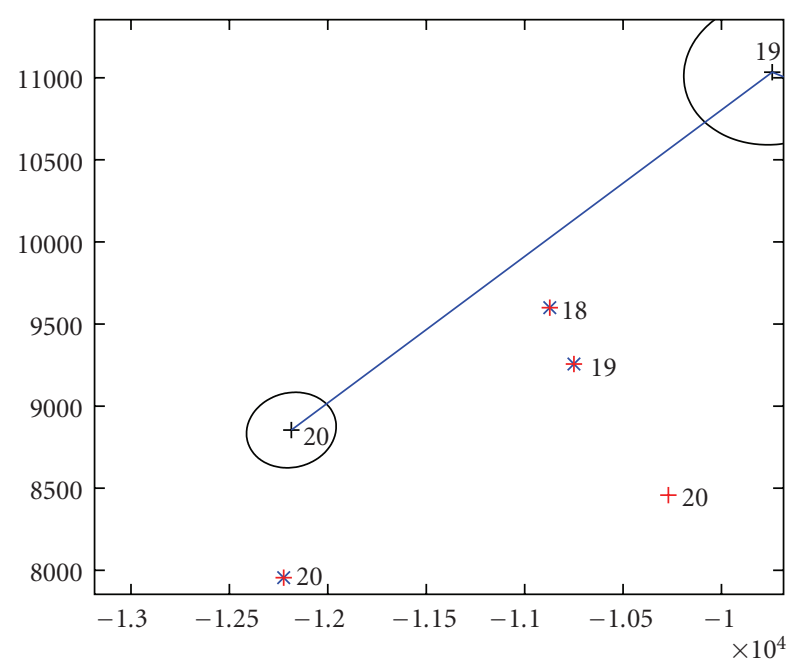

\begin{tabular}{r|r}
\multicolumn{1}{c|}{$t$} & \multicolumn{1}{|c}{$F_{1} \cdot F_{2}$} \\
\hline 4 & 3436.858 \\
5 & 4807.037 \\
$\mathbf{6}$ & $\mathbf{0 . 0 2 5}$ \\
7 & 1139.579 \\
8 & 2997.305 \\
$\mathbf{9}$ & $\mathbf{0 . 0 2}$ \\
10 & 276.007 \\
11 & 4556.095 \\
12 & 4497.803 \\
13 & 2354.912 \\
14 & 1126.75 \\
15 & 2991.504 \\
16 & 3647.314 \\
17 & 1806.277 \\
$\mathbf{1 8}$ & $\mathbf{0 . 0 1 9}$ \\
$\mathbf{1 9}$ & $\mathbf{0 . 0 8 1}$ \\
$\mathbf{2 0}$ & $\mathbf{3 . 3 6 1}$
\end{tabular}

(a)

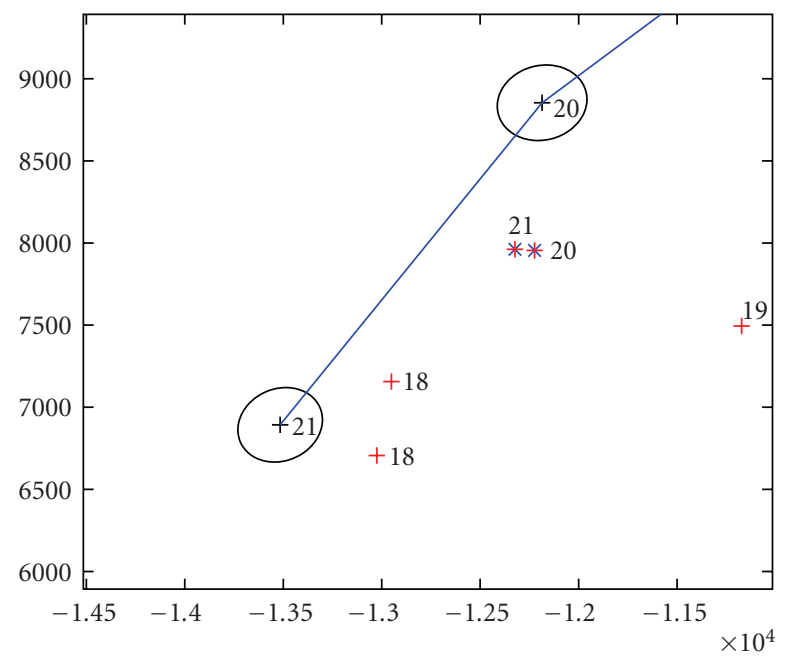

\begin{tabular}{r|r}
\multicolumn{1}{c|}{$t$} & \multicolumn{1}{|c}{$F_{1} \cdot F_{2}$} \\
\hline 5 & 4807.037 \\
$\mathbf{6}$ & $\mathbf{0 . 0 2 5}$ \\
7 & 1139.579 \\
8 & 2997.305 \\
$\mathbf{9}$ & $\mathbf{0 . 0 2}$ \\
10 & 276.007 \\
11 & 4556.095 \\
12 & 4497.803 \\
13 & 2354.912 \\
14 & 1126.75 \\
15 & 2991.504 \\
16 & 3647.314 \\
17 & 1806.277 \\
$\mathbf{1 8}$ & $\mathbf{0 . 0 1 9}$ \\
$\mathbf{1 9}$ & $\mathbf{0 . 0 8 1}$ \\
$\mathbf{2 0}$ & $\mathbf{3 . 3 6 1}$ \\
$\mathbf{2 1}$ & $\mathbf{0 . 1 1 4}$
\end{tabular}

(b)

Figure 6: Scan 20 and 21.

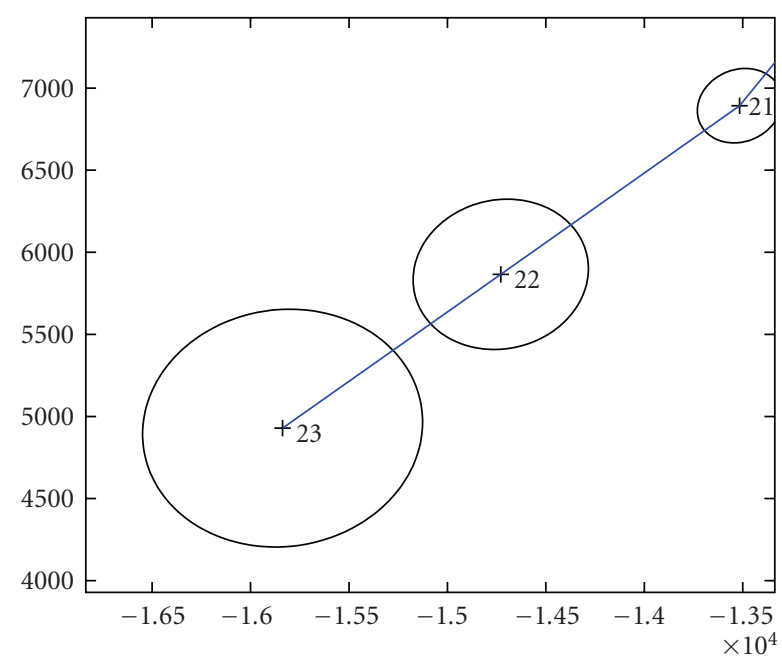

\begin{tabular}{r|r}
\multicolumn{1}{c|}{$t$} & \multicolumn{1}{|c}{$F_{1} \cdot F_{2}$} \\
\hline $\mathbf{6}$ & $\mathbf{0 . 0 2 5}$ \\
7 & 1139.579 \\
8 & 2997.305 \\
$\mathbf{9}$ & $\mathbf{0 . 0 2}$ \\
10 & 276.007 \\
11 & 4556.095 \\
12 & 4497.803 \\
13 & 2354.912 \\
14 & 1126.75 \\
15 & 2991.504 \\
16 & 3647.314 \\
17 & 1806.277 \\
$\mathbf{1 8}$ & $\mathbf{0 . 0 1 9}$ \\
$\mathbf{1 9}$ & $\mathbf{0 . 0 8 1}$ \\
$\mathbf{2 0}$ & $\mathbf{3 . 3 6 1}$ \\
$\mathbf{2 1}$ & $\mathbf{0 . 1 1 4}$ \\
$\mathbf{2 2}$ & $\mathbf{0 . 0 2 1}$ \\
$\mathbf{2 3}$ & $\mathbf{0 . 4 3 9}$
\end{tabular}

Figure 7: Scan 23. 


\subsection{Discussion of the example}

From scan 3 up to 5 there are neither missing detections nor disturbing impacts by false measurements. The synthetic measurement $\overline{\mathbf{z}}_{t}^{s}$ coincides quite well with the measurement that refers to the aircraft. Hence we can observe high values of $F_{1} \cdot F_{2}$ (Figure $2(\mathrm{a})$ ).

At scan 6, the aircraft was not detected. Hence $\overline{\mathbf{z}}_{t}^{s}=$ $(5114,5285)^{\top}$ is far away from $\mathbf{x}_{t \mid t-1}^{s}$ and has a large measurement error: $\overline{\mathbf{R}}_{t}^{s}(1,1)=\overline{\mathbf{R}}_{t}^{s}(2,2)=8.8017 \cdot 10^{23}$ and $\overline{\mathbf{R}}_{t}^{s}(2,1)=$ $\overline{\mathbf{R}}_{t}^{s}(1,2)=-2.1156 \cdot 10^{23}$. According to this, $F_{1} \cdot F_{2}$ is small $(<1)$.

Because of the missing detection at scan $t=6$, the prediction for scan 7 is bad, which is reflected by the blown up error ellipsoid (Figure 2(b)). As a consequence the prediction does not coincide with the synthetic measurement $\overline{\mathbf{z}}_{t}^{s}$ as well as it did during the preceding scans, and so the corresponding value of $F_{1} \cdot F_{2}$ is worse. At $t=8$, the PMHT recovered. But near the measurement of the aircraft, there is a false one. The synthetic measurement is the mean of both. It is farther away from the prediction than the measurement of the aircraft, and represents a different direction of movement. Thus $F_{1} \cdot F_{2}$ is smaller, compared with the scans $t=3,4,5$. This can be an indication for an impending lost of the track, because the estimations iteratively evolve a tendency towards the synthetic measurement. Therefore, the prediction at scan 9 sheers to the left (Figure 3 ).

At scan 9 the aircraft was again not detected and we observe the same effect as at scan 6: $F_{1} \cdot F_{2}<1$. Because of this missing detection in combination with the sheering at scan 8, the prediction for scan 10 is relatively bad. According to this-compared with the other scans the aircraft has been detected at - the value of $F_{1} \cdot F_{2}(276.007)$ is perspicuously worse.

At the scans 11 and 12 the track is again stable. The values of $F_{1} \cdot F_{2}$ approximately lie at 4500 (Figure 4(a)). And the false measurement at scan 13 again leads to a drift of the estimation. Therefore, the value of $F_{1} \cdot F_{2}$ for scan 14 amounts only to 1126.750 (Figure 4(b)). The PMHT again recovers at the scans 15 and 16 . In each case, the synthetic measurement coincides with the measurement of the aircraft. And the prediction hits this position quite well, as the product $F_{1} \cdot F_{2}$ reflects (Figure 5(a)).

At scan 17 the aircraft has not been detected. But a false measurement is picked up, that continues the track in an expedient way. At all further scans, the aircraft is also not detected. Its track ends at scan 16 (or at latest 17). According to this, $F_{1} \cdot F_{2}$ is relatively small $(<1)$ (Figures 6 and 7 ).

At scan 20 the synthetic measurement is (by chance) located in the further surrounding of the prediction. The consequence is a slight increase of $F_{1} \cdot F_{2}$ to 3.361 (Figure 6).

Table 1 shows all values of $F_{1} \cdot F_{2}$, recapitulating. Following [12] this value has to be multiplied with the previous LR at each scan (successive update). We see that $F_{1} \cdot F_{2}$ can be of a relatively high magnitude, which causes a fast increase while successively updating the LR. A possible means to reduce the magnitude could be the use of logarithmic values. But this idea has to be checked quite carefully because the logarithm becomes negative in case of values that are smaller than unity.
TABLE 1: LR calculation for a track of a single target.

\begin{tabular}{lccc}
\hline$t$ & $F_{1} \cdot F_{2}$ & $t$ & $F_{1} \cdot F_{2}$ \\
\hline 3 & 124307.556 & 15 & 2991.504 \\
4 & 3436.858 & 16 & 3647.314 \\
5 & 4807.037 & 17 & 1806.277 \\
$\mathbf{6}$ & $\mathbf{0 . 0 2 5}$ & $\mathbf{1 8}$ & $\mathbf{0 . 0 1 9}$ \\
7 & 1139.579 & $\mathbf{1 9}$ & $\mathbf{0 . 0 8 1}$ \\
8 & 2997.305 & $\mathbf{2 0}$ & $\mathbf{3 . 3 6 1}$ \\
$\mathbf{9}$ & $\mathbf{0 . 0 2 0}$ & $\mathbf{2 1}$ & $\mathbf{0 . 1 1 4}$ \\
10 & 276.007 & $\mathbf{2 2}$ & $\mathbf{0 . 0 2 1}$ \\
11 & 4556.095 & $\mathbf{2 3}$ & $\mathbf{0 . 4 3 9}$ \\
12 & 4497.803 & $\mathbf{2 4}$ & $\mathbf{0 . 1 1 6}$ \\
13 & 2354.912 & $\mathbf{2 5}$ & $\mathbf{0 . 0 1 9}$ \\
14 & 1126.750 & - & - \\
\hline
\end{tabular}

However, the proposed test function affords track extraction, reconfirmation and deletion within a PMHT track management framework, analogously to the traditional MHT approach $[12,19]$.

\section{CONCLUSIONS}

For PMHT, a solution to the problem of track extraction and deletion inspired in [12] has been proposed.

We presented an LR update formula for track extraction by PMHT. Using this formula the sequential LR test is a byproduct of the PMHT iteration process on the current window. All ingredients of the formula are calculated by PMHT. In a simulation the new formula was quantitatively discussed scan by scan.

The test function is applicable within a general track management framework, as it is presented in [7]. If we decide for track extraction, we can immediately switch to track maintenance. An open question in this context is the appropriate choice of the bounds $A$ and $B$. Finally, the computational load of our approach will be an important topic.

\section{APPENDIX}

\section{A. PRODUCT FORMULA FOR GAUSSIANS}

The product formula transforms a product of Gaussians into another product of Gaussians. There are two versions.

\section{(1) Version 1:}

$$
\mathcal{N}(\mathbf{x} ; \mathbf{X y}, \mathbf{Y}) \mathcal{N}(\mathbf{y} ; \mathbf{z}, \mathbf{Z})=\mathcal{N}(\mathbf{x} ; \mathbf{a}, \mathbf{A}) \mathcal{N}(\mathbf{y} ; \mathbf{b}, \mathbf{B})
$$

with

$$
\begin{aligned}
& \mathbf{a}=\mathbf{X z}, \\
& \mathbf{A}=\mathbf{X Z X}^{\top}+\mathbf{Y} \text {, } \\
& \mathbf{b}=\mathbf{z}+\mathbf{W}(\mathbf{x}-\mathbf{X z}), \\
& \mathbf{B}=\mathbf{Z}-\mathbf{W A W}^{\top} \text {, } \\
& \mathbf{W}=\mathbf{Z X}^{\top} \mathbf{A}^{-1} \text {. }
\end{aligned}
$$




\section{(2) Version 2:}

$$
\mathcal{N}(\mathbf{x} ; \mathbf{y}, \mathbf{Y}) \mathcal{N}(\mathbf{x} ; \mathbf{z}, \mathbf{Z})=\mathcal{N}(\mathbf{x} ; \mathbf{a}, \mathbf{A}) \mathcal{N}(\mathbf{z} ; \mathbf{b}, \mathbf{B})
$$

with

$$
\begin{aligned}
& \mathbf{a}=\mathbf{A}\left(\mathbf{Y}^{-1} \mathbf{y}+\mathbf{Z}^{-1} \mathbf{z}\right), \\
& \mathbf{A}=\left(\mathbf{Y}^{-1}+\mathbf{Z}^{-1}\right)^{-1}, \\
& \mathbf{b}=\mathbf{y} \\
& \mathbf{B}=\mathbf{Y}+\mathbf{Z} .
\end{aligned}
$$

\section{REFERENCES}

[1] S. S. Blackman and R. Populi, Design and Analysis of Modern Tracking Systems, Artech House, Boston, Mass, USA, 1999.

[2] Y. Bar-Shalom and T. E. Fortmann, Tracking and Data Association, Academic Press, New York, NY, USA, 1988.

[3] R. L. Streit and T. E. Luginbuhl, "Probabilistic multihypothesis tracking," Tech. Rep. NUWC-NPT/10/428, Naval Undersea Warefare Center Division, Newport, RI, USA, February 1995.

[4] R. L. Streit, "The PMHT and related applications of mixture densities," in Proceedings of the 9th International Conference on Information Fusion (FUSION '06), Florence, Italy, July 2006.

[5] F. Dellaert, "The expectation maximization algorithm," Tech. Rep. GIT-GVU-02-20, College of Computing, Georgia Institute of Technology, Atlanta, Ga, USA, 2002, http://www-static.cc.gatech.edu/gvu/reports/2002/abstracts/ 02-20.html.

[6] M. A. Tanner, Tools for Statistical Inference, Springer, New York, NY, USA, 1996.

[7] S. J. Davey and D. A. Gray, "Integrated track maintenance for the PMHT via the hysteresis model," IEEE Transactions on Aerospace and Electronic Systems, vol. 43, no. 1, pp. 93-111, 2007.

[8] T. E. Luginbuhl, Y. Sun, and P. K. Willett, "A track management system for the PMHT," in Proceedings of the Conference on Data Fusion, Montreal, Canada, August 2001.

[9] C. G. Hempel and S. L. Doran, "A PMHT algorithm for active sonar," in Acquisition, Tracking, and Pointing XVIII, vol. 5430 of Proceedings of SPIE, pp. 132-142, Orlando, Fla, USA, April 2004.

[10] S. J. Davey, Extensions to the probabilistic multi-hypothesis tracker for improved data association, Ph.D. thesis, University of Adelaide, Adelaide, Australia, 2003.

[11] S. J. Davey and D. A. Gray, "A comparison of track initiation methods with the PMHT," in International Conference on Information, Decision and Control, pp. 323-328, Adelaide, Australia, February 2002.

[12] G. Van Keuk, "Sequential track extraction," IEEE Transactions on Aerospace and Electronic Systems, vol. 34, no. 4, pp. 11351148, 1998.

[13] H. Gauvrit, J. P. Le Cadre, and C. Jauffret, "A formulation of multitarget tracking as an incomplete data problem," IEEE Transactions on Aerospace and Electronic Systems, vol. 33, no. 4, pp. 1242-1257, 1997.

[14] P. K. Willett, Y. Ruan, and R. L. Streit, "PMHT: problems and some solutions," IEEE Transactions on Aerospace and Electronic Systems, vol. 38, no. 3, pp. 738-754, 2002.

[15] Y. Ruan and P. Willett, "The turbo PMHT," IEEE Transactions on Aerospace and Electronic Systems, vol. 40, no. 4, pp. 13881398, 2004.
[16] M. Wieneke and W. Koch, "The PMHT: solutions for some of its problems," in Signal and Data Processing of Small Targets, vol. 6699 of Proceedings of SPIE, pp. 1-12, San Diego, Calif, USA, August 2007.

[17] C. Rago, P. K. Willett, and R. L. Streit, "A modified PMHT," in Proceedings of the Conference on Information Sciences and Systems, Baltimore, Md, USA, March 1995.

[18] A. Wald, Sequential Analysis, John Wiley \& Sons, New York, NY, USA, 1947.

[19] G. Van Keuk, "MHT extraction and track maintenance of a target formation," IEEE Transactions on Aerospace and Electronic Systems, vol. 38, no. 1, pp. 288-295, 2002.

[20] W. Koch, "Sensor data fusion: methods, applications, examples," in Advances and Challenges in Multisensor Data and Information Processing, E. Lefebre, Ed., NATO Security through Science, IOS Press, Amsterdam, The Netherlands, April 2007. 